\title{
Application of Digital Signal Processing In Echo Cancellation: A Survey
}

\author{
D. Jalaputhra \\ Faculty of Electronic and \\ Computer Engineering \\ Universiti Teknikal Malaysia \\ Melaka (UTeM)
}

\author{
A.S. Ja'afar \\ Faculty of Engineering \\ Technology \\ Universiti Teknikal Malaysia \\ Melaka (UTeM)
}

\author{
N. M. Z. Hashim \\ Faculty of Electronic and \\ Computer Engineering \\ Universiti Teknikal Malaysia \\ Melaka (UTeM) \\ A.Salleh \\ Faculty of Electronic and \\ Computer Engineering \\ Universiti Teknikal Malaysia \\ Melaka (UTeM)
}

\author{
M. Izhan Ibrahim \\ Faculty of Electronic and \\ Computer Engineering \\ Universiti Teknikal Malaysia \\ Melaka (UTeM) \\ N. R. Mohamad \\ Faculty of Electronic and \\ Computer Engineering \\ Universiti Teknikal Malaysia \\ Melaka (UTeM)
}

\begin{abstract}
The advanced communications world is worried talking more naturally by using hands free this help the human being to talk more confidently without holding any of the devices such as microphones or telephones. Acoustic echo cancellation and noise cancellers are quite interesting nowadays because they are required in many applications such as speakerphones and audio/video conferencing. This paper describes an alternative method of estimating signals corrupted by additive noise or interference. Acoustic echo cancellation problem was discussed out of different noise cancellation techniques by concerning different parameters with their comparative results. The results shown are using some specific algorithms.
\end{abstract}

Keywords: Acoustic Echo Cancellation (AEC), Adaptive Filtering, Algorithm, Hybrid, Noise Cancellers.

\section{INTRODUCTION}

The growth of the cellular phone market in the last years has led to an increase on the quality of handset receivers. In particular, the quality of the audio is one of the features the cellular vendors take in high consideration. One issue related to the audio quality is the need for an acoustic echo suppression device which eliminates the far speech signal that propagated between microphones and loudspeaker. Nowadays, the user around the world had used the speaker phones and hands-free phones for their video teleconferencing and audio-conferencing in their daily life and business. It will allow full-duplex communication without having to hold the phone which the data can flow two ways at the same time. Full duplex devices also can communicate back and forth simultaneously.

The existence of echo signal would make conversation difficult. There are several echo signals generated from various factors such as digital network echo and $\mathrm{AE}$ (acoustic echo) signal. Most of all, the AE generated between loud-speaker signal and near-end speaker signal, affects the conversation quality significantly, for hands-free set the effective AEC (acoustic echo canceller) is required for better performance. NLMS based the adaptive filtering algorithm is used for AEC because the statistical characteristics of the input signal of the AEC and the echo path are change by time 1. A big reason that is this AEC algorithm for the hands-free fix must operate in real time.

The repetition of sound is called echo which the speech from the far-end caller is transmitted by the speakerphone and transmitted again itself by bouncing off the inside surface. Existence of feedback loop where the far-end caller hears some echo of his own voice is because echoes are picked up by the near-end microphone. One of the effective techniques to reduce this problem is by using the digital signal processing technique as known as acoustic echo cancellation
(AEC) or telephone line hybrid echo which it will prevent the feedback and at the same time it will allow full-duplex communication.

Echo can be defined as a waveform which repeated due to reflection from points where the characteristics of the medium through the transmitted wave. It is very useful for the detection and exploration of sonar and radar. In telecommunication system, echo will be influence the quality service. Echo is speech reflection causing the interferences.

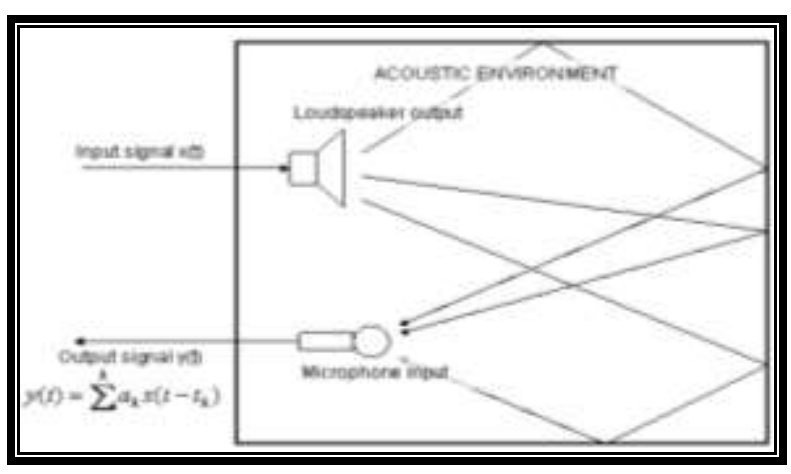

Figure 1: Origin of acoustic echo

In the year 1950's efforts to reduce the echo was carried out by dividing into two types which of acoustic echo and telephone line hybrid echo. As far as possible, it is very important about the echo cancellation because it will increase the quality service in communication service.

\section{TYPES OF ECHO}

There are two types of echo might be classified with telecommunication which are Acoustic Echo and Line Hybrid Echo. 


\subsection{Acoustic Echo}

A acoustic echo can be explained as sound trap against microphones, then grab sound indicators from your speaker, along with transmits this returning to the originating user. The particular originating user will then hear the particular echo in the user's personal style for the reason that participator echoes.

Acoustic echo is usually become more sensitive as soon as microphones are utilized, and also either the speaker or mike sound system is turned up to as well as if your mike along with speakers is situated so the mike is close to several in the speakers. This specific echo is annoying by simply reflective acoustic echo reflected by simply surfaces and/or physical objects.

Acoustic echo may be caused or made worse while extremely sensitive microphones are utilized, speaker volume is turned up quite high, or the particular microphone along with speaker are extremely close up to each other. So, this may interfere the conversation between participants due to the annoying echo.

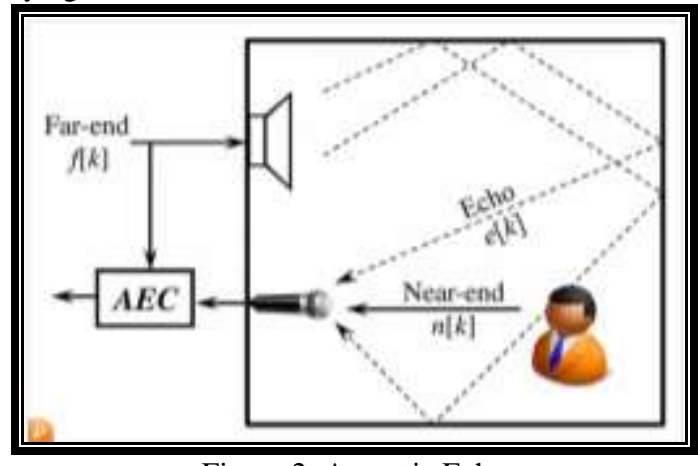

Figure 2: Acoustic Echo

\subsection{Hybrid}

Hybrid - Essentially the most frequent root cause of indicate in PSTN is impedance1 mismatches in the cross in which 4-wire cell phone circuits are usually transformed into 2-wire circuits. This electrically produced indicate happens if your inward bound vitality in the much end loudspeaker is reflected rear when it comes to your loudspeaker to be a a little bit improved in addition to detained duplication caused by impedance mismatch from the cross. The actual reputation connected with indicate happens each time your replicated signal wait is greater than 10 milliseconds in addition to becomes apparent on the loudspeaker as reflected voice if your wait is greater than as small as sixteen msec. It truly is described on the much end as an improved imitation with the loudspeaker initial.

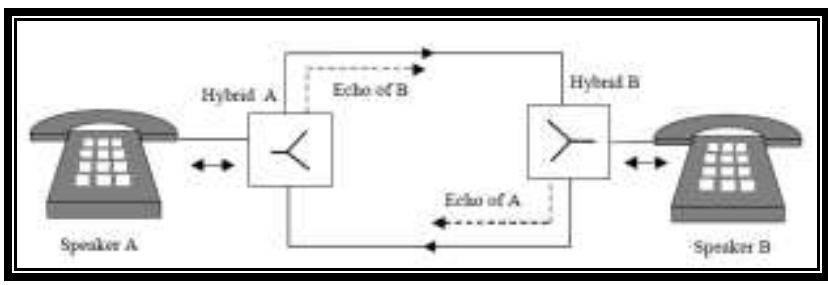

Figure 3: Some of the an cellular call by coverage linked with 2-wire subscriber's using hybrids to 4-wire outlines on the trade

Active hybrid circuits provide a few echo decreases, though not sufficient if the end-to-end circuit delay is even moderate. The network being reflected back is measured as ERL (Echo Return Loss), the greater the ERL, the lesser the reflected signal back to the speaker. Impedance: the ratio of the electromotive force effective to efficient echo is contrary currents in the electrical circuit for (AC) alternating current to (DC) direct current.

\section{ECHO REDUCTION TECHNIQUES}

In this section a general survey of echo cancelling technique is presented:

\subsection{Adaptive Filtering}

Normally when echo appears adaptive filter is typically been used to overcome the noise whether known or repetition of echo happens. Diagram below shows the basic form of time-domain adaptive filtering application in echo cancellation.

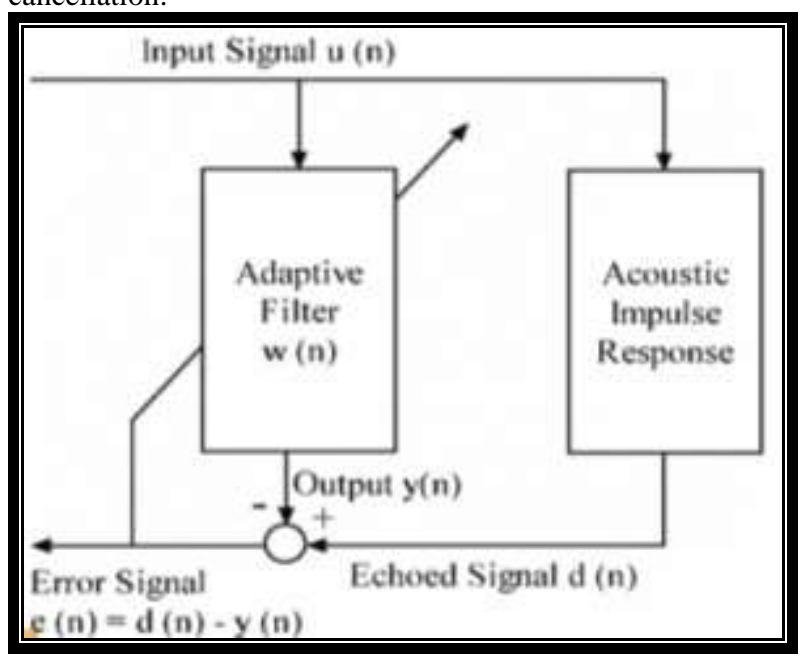

Figure 4: Adaptive Echo Cancellation block diagram

\subsection{Principle of Echo Cancellation}

The typical ways of echo cancellation are primarily according to the identification of the acoustic channel. This channel is commonly modelled by a finite impulse response (FIR) filter with length This linear modelling could be justified by understanding that the channel is , initially approximation , com posed basically of delay and attenuation - The longer the valuable period assist of the impulse response, the larger is the length $\mathrm{L}$ needed for modelling. In practice, this time support can vary from several dozen milliseconds (vehicle compartment) to several hundred milliseconds (conference room).

The echo cancellation algorithm enables filter to be estimated by the L-size vector $\mathrm{h}(\mathrm{k})$ using a criterion based on the a priori estimation error. This estimation error, called residual echo, is written, for each sample $\mathrm{k}$.

$$
e(k)=y(k)-\mathbf{h}^{T}(k) \mathbf{x}(k)
$$

where $\mathbf{x}(k)=[x(k), x(k-1), \ldots, x(k-L+1)]^{T}$

The L last samples of the loudspeaker signal. The filter is updated at each instant by feedback of the estimation error proportional to the adaptation gain, denoted as $\mathrm{c}(\mathrm{k})$, and according to

$$
\mathbf{h}(k+1)=\mathbf{h}(k)-\mathbf{c}(k))(k)
$$


The different echo cancellation algorithms are distinguished by the gain calculation $\mathrm{c}(\mathrm{k})$.These algorithms can be classified as follows

(i) Algorithms produced from the gradient least mean squares in which the optimization criterion correspondents to a reduction of the mean-square error.

(ii) ( RLS ) Recursive least squares algorithms are depending on a reduction of the criterion of the least squares with exponential forgetting where is a forgetting factor.

$$
f[\mathbf{h}(k)]-\sum_{i=0}^{k} \lambda^{k-i}\left[y(i)-\mathbf{h}^{T}(i) \mathbf{x}(i)\right]^{2}
$$

Moreover, the predictable part of the input signal can be extracted with predictors of lower order than the filter size leading to a class of Newton-type algorithms known as fast Newton transversal filters.

\section{ACOUSTIC ECHO CANCELLATION}

Block diagram below shows the structure of the AEC adaptive filtering.

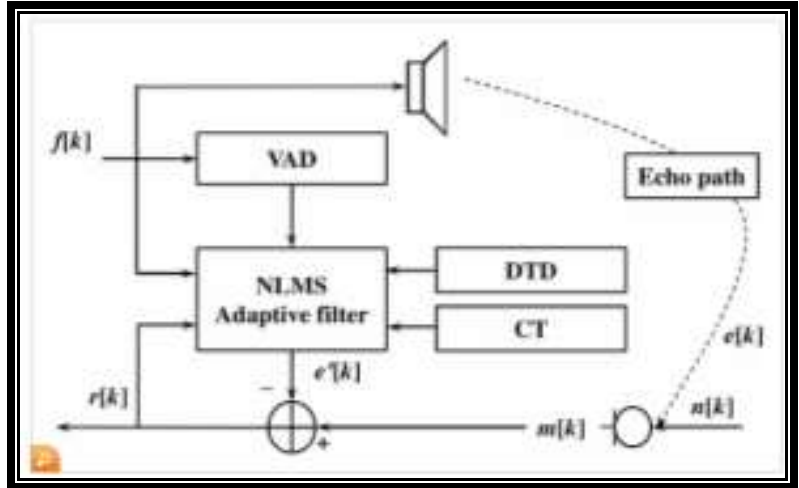

Figure 5: Block Diagram of proposed AEC

A good canceller its performe during the DT it is one of the important characteristics.

$$
\begin{array}{ll}
p[k+1]=\left(1-\alpha_{\text {high }}\right) p[k]+\alpha_{\text {high }} f[k]^{2} \geq \gamma, & p[k] \geq p[k-1] \\
p[k+1]=\left(1-\alpha_{\text {low }}\right) p[k]+\alpha_{\text {low }} f[k]^{2} \geq \gamma, & p[k]<p[k-1]
\end{array}
$$

In order to overcome the problem of the AEC, a proper methodology to be designed to filter out the Acoustic Echo Cancellation. A flow chat above shows the methodology proposed for the Acoustic Echo Cancellation.

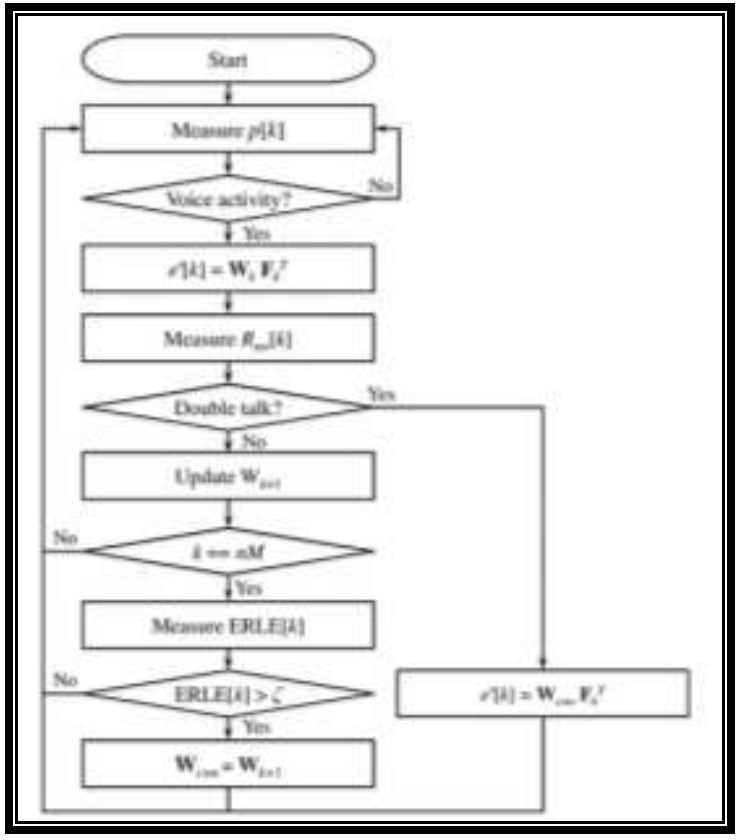

Figure 4 Proposed AEC

\subsection{Types of Noise Signals}

Five types of common signal that can be interrupted an acoustic environment as shown in table below. By using Acoustic Noise Canceller system all kind of noise able to remove.

Table 1: Varieties of Noise Signal

\begin{tabular}{|c|c|}
\hline $\begin{array}{c}\text { Types of } \\
\text { Noise }\end{array}$ & Description \\
\hline Violet & $\begin{array}{c}\text { Frequency premphasis } 6 \mathrm{~dB} \\
\text { intensity characteristic of } \mathrm{f}^{\wedge} 2 \\
\text { inverse of brown noise }\end{array}$ \\
\hline Blue & Sense of inverse pink noise \\
\hline Brown & Kind of noise with random walks \\
\hline Pink & The most prevalent noise in nature \\
\hline White & Flat response \\
\hline
\end{tabular}

\section{CONCLUSION}

In this paper clearly explained the method of Acoustic Echo cancellation specifically chosen to enhance the quality of the conversation especially for hands-free set. Additionally adaptive filter proposed to satisfy the demanded echo reduction furthermore the missed DT was compensate during voice growing time. Implementing the adaptive filter in the frequency domain, on the other hand, reduces the computation complexity and improves the AEC performance. Last but not list the proper methodology been designed to propose the Acoustic echo cancellation.

\section{ACKNOWLEDGMENTS}

We are grateful to Centre for Telecommunication Research and Innovation (CeTRI) and Universiti Teknikal Malaysia Melaka (UTeM) through PJP/2013/FKEKK (29C)/S01215 for their kind and help for supporting financially and supplying the electronic components and giving their laboratory facility to complete this study. 


\section{REFERENCES}

[1] Halder, Patrashiya Magdolina, and AKM Fazlul Haque. "Improved Echo cancellation in VOIP." Dept. of Electronics and Telecommunication Engineering, Daffodil International University, Dhaka, Bangladesh, International Journal of Advanced Computer Science and Applications 2.11 (2011).

[2] http://www.pikatechnologies.com/english/View.asp?mp= $\underline{825 \& \mathrm{x}=832}$

[3] http://www.voipunlimitedcalls.com/the-use-of-voip-echocancellation/

[4] http://www.adaptivedigital.com/product/echo_cancel/echo explain.htm

[5] N. M. Z. Hashim, A. F. Jaafar, Z. Zakaria, A. Salleh, and R. A. Hamzah, "Smart Casing for Desktop Personal Computer," International Journal of Engineering and Computer Science (IJECS), vol. 2, no. 8, pp. 2337-2342, 2013.

[6] N. M. Z. Hashim, N. H. Mohamad, Z. Zakaria, H. Bakri, and F. Sakaguchi, "Development of Tomato Inspection and Grading System using Image Processing," International Journal Of Engineering And Computer Science (IJECS), vol. 2 no. 8, pp. 2319-2326, 2013.

[7] N. M. Z. Hashim, N. A. Ali, A. Salleh, A. S. Ja'afar, and N. A. Z. Abidin, "Development of Optimal Photosensors Based Heart Pulse Detector," International Journal Of Engineering and Technology (IJET), vol. 5, no. 4, pp. 3601-3607, 2013.

[8] N. M. Z. Hashim, N. M. T. N. Ibrahim, Z. Zakaria, F. Syahrial, and H. Bakri, "Development New Press Machine using Programmable Logic Controller," International Journal of Engineering and Computer Science (IJECS), vol. 2, no. 8, pp. 2310-2314, 2013.

[9] N. M. Z. Hashim, N. A. Ibrahim, N. M. Saad, F. Sakaguchi, and Z. Zakaria, "Barcode Recognition System," International Journal of Emerging Trends \& Technology in Computer Science (IJETTCS), vol. 2, no. 4, pp. 278-283, 2013. 